\title{
Article
}

\section{Accuracy of relational and featural information in facial-composite images}

Fodarella, Cristina and Frowd, Charlie

Available at http://clok.uclan.ac.uk/22023/

Fodarella, Cristina ORCID: 0000-0001-5551-3450 and Frowd, Charlie ORCID: 0000-0002-5082-1259 (2013) Accuracy of relational and featural information in facial-composite images. Emerging Security Technologies (EST), 2013 Fourth International Conference on Emerging Security Technologies .

It is advisable to refer to the publisher's version if you intend to cite from the work. http://dx.doi.org/10.1109/EST.2013.9

For more information about UCLan's research in this area go to

http://www.uclan.ac.uk/researchgroups/ and search for <name of research Group>.

For information about Research generally at UCLan please go to http://www.uclan.ac.uk/research/

All outputs in CLoK are protected by Intellectual Property Rights law, including Copyright law. Copyright, IPR and Moral Rights for the works on this site are retained by the individual authors and/or other copyright owners. Terms and conditions for use of this material are defined in the policies page. 


\section{Accuracy of Relational and Featural Information in Facial-Composite Images}

\author{
Cristina Fodarella \\ School of Psychology \\ University of Central Lancashire \\ Preston, UK \\ cfodarella@uclan.ac.uk
}

\author{
Charlie D. Frowd \\ Department of Psychology \\ The University of Winchester \\ Winchester, UK \\ charlie.frowd@winchester.ac.uk
}

\begin{abstract}
Facial-composite images are crucial in helping to bring perpetrators to justice and thereby maintain national and international security. Psychology can assist in identifying the accuracy of such facial information as well as ways to improve their identification rates. The current experiment demonstrates that composites tend to differ in terms of their accuracy of featural information (individual facial features) and relational information (spacing between those facial features) depending on the type of composite software used. Composites constructed with feature systems tend to have more-accurate feature information, whilst those constructed with holistic systems tend to have more-accurate relational information. Further, it was found that not only the addition of sunglasses on poor-quality composites, but also viewing composites from the side, can increase their identifiability. This could be applied by the police to potentially increase identification and arrest rates of perpetrators.
\end{abstract}

Keywords - facial composite; psychology; face recognition; featural information; configural information; perceptual stretch

\section{INTRODUCTION}

Face perception is considered to be an inborn and predispositional capacity [1] enabling processing of the great complexity of a face. Familiar faces are recognised extremely fast [2] and accurately in a variety of visual angles, lighting or other changes [3] - even when a photograph of the particular face is of poor quality [4]. Familiar faces are better recognised on the basis of their internal features (e.g. eyes, nose, mouth) rather than their external features (e.g. hair, ears) [5]. The eye region in particular seems to be extremely important for identifying familiar faces [6]. However, not only are individual features crucial for recognition, but also the spatial arrangements of these features on the face [7]. Here, we refer to the physical distances between these facial features as configural information.

It seems likely that the brain applies both featural- and configural-based strategies for identifying faces, and is capable of switching between those types depending on the task required [8]. To investigate the role of featural and configural information in face perception, photographs of faces can be manipulated to examine their recognisability. Manipulations are typically affine and include inversion, blur, stretch, photographic negation and caricature.

Inversion is a $180^{\circ}$ picture-plane rotation, which greatly disrupts face recognition [9-11]. It is suggested that the processing of configural information becomes largely impossible with inversion [12,13] and that faces are recognised principally on the basis of their individual facial features [14-17]. In general, it seems that configural processing is particularly important for face recognition.

In contrast to inversion, Gaussian or blur (low pass) filtering of facial images is considered to have the opposite effect as it conceals individual features [18]. Although recognition performance declines, faces seen with even fairlyhigh levels of blur remain identifiable [19], implying that the brain uses a configural processing strategy for identification [20]. Inversion and blur in combination have been shown to render face recognition impossible as both featural and configural information cannot be processed $[21,22]$.

A further image manipulation is linear stretch. Although it leads to changes in both configural and feature information, stretched photographs surprisingly remain as recognisable as veridical ones [19]. It was suggested that it is the use of configural information that enables this recognition [19].

The recognition of faces is especially important within a forensic context. In a crime, victims and witnesses are often required to remember and recall a perpetrator's face in order to create a facial composite. This is a facial likeness constructed from memory via appropriate computer software or a sketch artist. The facial composite would then be circulated within a police force or published more widely in the media in the hope that someone will correctly identify the perpetrator, enabling their arrest.

There are two contrasting systems for creating composites, feature and holistic. In the former case, witnesses are presented with a range of individual feature features which can be selected and altered until accomplishing a likeness of the perpetrator. In the latter system, witnesses are shown sets of 
whole faces and asked to select those that are overall most similar to the face they had seen. Selected faces are combined, enabling the software to 'breed' further sets of faces. This procedure is repeated until a face is 'evolved'. Subsequently, shape and placement of facial features along with holistic characteristics (e.g. age, weight, health, etc.) can be altered. There are a number of different feature and holistic systems available. For example, popular feature systems are PRO-fit and E-FIT in the UK and Europe, and Identikit 2000 and FACES in the US. In contrast, popular holistic systems are EvoFIT and EFIT-V in the UK, and ID in South Africa [23].

In Section 2, we apply image manipulations to composites from those two types of system to reveal potential differences in accuracy of featural and configural information. Not only because it is extremely difficult for witnesses to convey what the perpetrators looked like, but also due to normal fading of memory, facial composites lack accuracy [24]. Therefore, we also aim to identify ways of improving the identification of composites which could potentially be of poor likeness.

\section{EXPERIMENT}

A recent study [25] was the first to apply these manipulations on facial composites to explore the accuracy of their featural and relational information. In that study, facial composites were of famous faces, as these type of celebrity images had also been used in the literature for manipulations with facial photographs. Participant-witnesses were familiar to these faces before they constructed the composites. There was typically a short, 3-4 hour delay between their viewing the target face and constructing the facial composite with either a feature (PROfit or E-FIT) or a holistic system (EvoFIT). Composites were then manipulated with different degrees of blur, inversion, linear stretch and photographic negation.

Their findings revealed that whilst both feature and holistic composites contained accurate featural and configural information to some extent, very high-detailed feature information was more accurate in feature composites but less accurate in holistic composites. It seems likely that the differences in the way the composites had been constructed led to these findings: people selected individual features (eyes, nose, mouth) in feature systems and in EvoFIT they were asked to select faces on the basis of their overall appearance.

Frowd et al reported a rather unexpected finding: linear stretch increased recognisability of the composites. It was theorised that this manipulation might help overcoming inaccurate aspects which are naturally present in composites. The beneficial effect was subsequently replicated with a 'perceptual' rather than 'physical' stretch. In the former case, participants looked at the composite from the side, by turning the image on a printed page, to give the appearance of a stretched face.

Here, we aim to replicate Frowd et al's findings using more-realistic, ecologically valid procedures; these should, therefore, give results that are more applicable to real life. Specifically, we use non-familiar target faces, as witnesses in real life are more likely to be unfamiliar to the perpetrator. To do this, participants were initially screened so as to construct a composite of an unfamiliar target. Also, we use a longer delay between viewing of the target faces and construction of the composites: in a real life situation, witnesses often construct a composite one or two days after a crime has occurred. A 24hour rather than a 48-hour delay was chosen mainly for convenience, but also because a comparison of four studies revealed only $2 \%$ difference in correct naming scores of composites across 24- to 48-hour delays (see [23]).

Materials: Twenty-four facial composites were constructed and then manipulated by adding inversion, Gaussian blur (at 8 cycles per face width), inversion-and-blur, and sunglasses. The final manipulation, with sunglasses added, was chosen as past research has found that poor-quality composites became more-recognisable with sunglasses added, indicating the ineffectiveness of this region of the face with this type of composite. Also, consistent with this idea, the opposite was found for good quality composites, which became lessrecognisable with sunglasses [26]. This result is in line with findings highlighting the importance of the eye region when recognising familiar faces [6]. Examples are shown in Fig 1. Adobe Photoshop was used to apply blurring and add sunglasses, whilst Microsoft Word was used for inversion.

Testing procedure: The testing procedure was carried out in two sections, and each involved a separate group of participants. Firstly, the composites were constructed and secondly, the composites were named.

For composite construction, 24 participants were shown an unfamiliar target photograph of a UK footballer (Andy Carroll, Fernando Torres, Frank Lampard, Gareth Barry, James Milner, John Terry, Michael Carrick, Peter Crouch, Robin van Persie, Ryan Giggs, Scott Parker and Steven Gerrard) for 60 seconds, and asked to construct a facial composite after a 24-hour delay. Half were assigned to the PRO-fit feature system and half to the EvoFIT holistic system.

In the composite naming task, 100 adult visitors to the National Football Museum (Manchester) were asked to name either veridical composites or composites that had been blurred, inverted, inverted-and-blurred, or were wearing sunglasses. These composites were presented randomly and were named twice: participants viewed the composites from

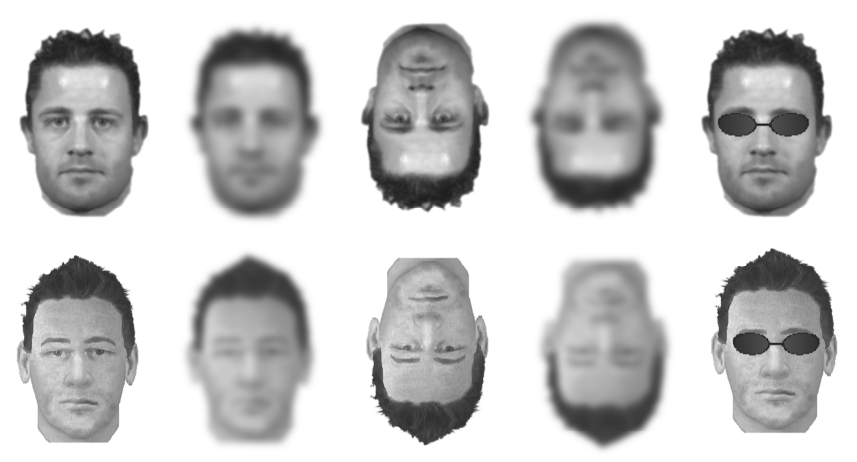

Fig. 1. Example composites used in the study. They are an EvoFIT of James Milner (row 1) and a PRO-fit of John Terry (row 2). Manipulations were (left to right): Veridical, Blur, Inversion, Inversion-and-Blur, and Sunglasses. 
the front, and then (unexpectedly for the participants) from the side, enabling a perceptual stretch. To ensure that participants were familiar with the target identities, original photographs were then shown for participants to identify. Participants were tested individually and the task was self-paced.

Results: Familiarity of the target identities was very high, at $98 \%$ overall. Correct naming of composites was analysed to investigate differences for system, that is between feature and holistic system; view, that is front and side view; and presentation, that is veridical, blur, inversion, inversion-andblur, and sunglasses. Fig. 2 demonstrates the main effect of view $(p<0.01)$ with composites being named significantly better when viewed from the side than from the front. Further, there was a significant main effect of presentation $(p<0.001)$, and two-tailed simple contrasts revealed that, relative to veridical composites, inverted and inverted-and-blurred composites were named significantly worse $(p<0.001)$.

There were two significant interactions. The first was between view and presentation $(p<0.05)$ (see also Fig. 2). Two-tailed simple contrasts relative to veridical revealed that inverted and inverted-and-blurred composites were named significantly worse regardless of view type $(p<0.05)$. Further, paired sample t-tests demonstrated that only veridical composites were named significantly better when viewed from the side than from the front $(p<0.05)$.

Fig. 3 illustrates the second significant interaction, between system and presentation $(p<.001)$. Two-tailed simple contrasts relative to veridical revealed that inversion and inversion-and-blur significantly decreased naming scores of EvoFITs, whilst blur and inversion-and-blur decreased those for PRO-fits $(p<.05)$. PRO-fits with sunglasses were named significantly better than veridical PRO-fits $(p<0.01)$.

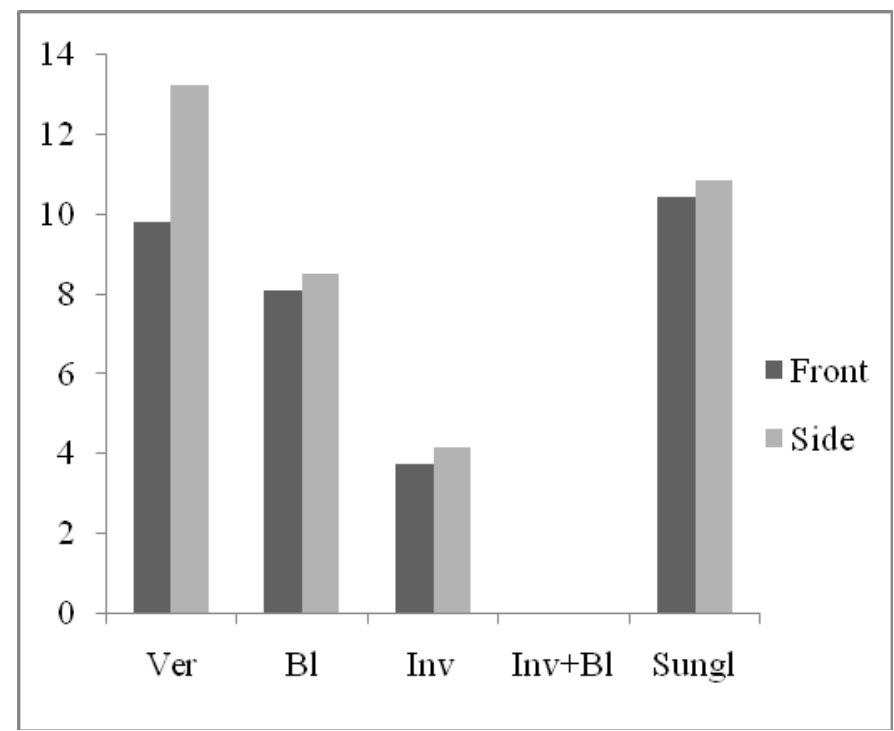

Fig. 2. Percentage-correct naming scores for combined holistic and feature composites across view type and presentations (left to right): Veridical, Blur, Inversioin, Inversion-and-Blur, and Sunglasses.

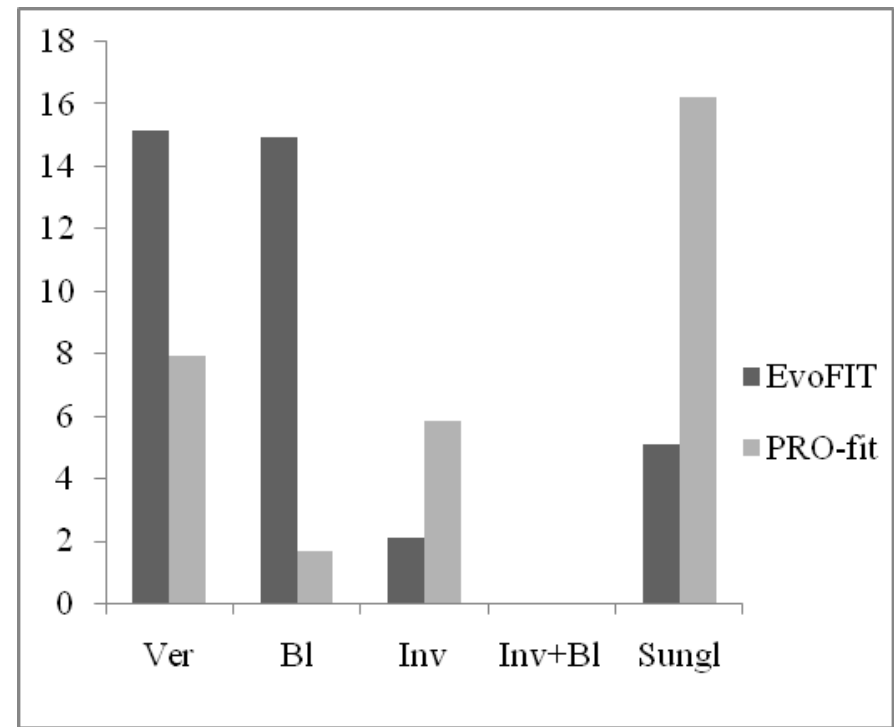

Fig. 3. Percentage-correct naming scores from both views combined for EvoFIT (holistic) and PRO-fit (feature) composites across presentations (left to right): Veridical, Blur, Inversion, Inversion-and-Blur, and Sunglasses.

Furthermore, inversion $(p<.05)$ and sunglasses $(p<.001)$ led to PRO-fits being more recognisable than EvoFITs, and blur $(p<.05)$ led to EvoFITs being better named than PRO-fits.

An analysis of incorrect composite naming was run to consider participants' willingness to attempt identification. It was found that composites were incorrectly named to the same degree across all manipulations, systems and views. Whilst incorrect naming percentages in past studies were around $20 \%$ [24], or 30\% [26], in the current experiment they were rather low, at $8 \%$. (See Section 3 for interpretation of this result.)

\section{DISCUSSION}

The experiment investigated differences in accuracy of featural and configural information in composites constructed with feature and holistic systems. Also, ways of improving the recognisability of composites were explored. Participantwitnesses viewed target faces and constructed composites with either EvoFIT or PRO-fit after a 24-hour-delay. Both sets of composites were subsequently manipulated by inversion, blur, inversion-and-blur, and adding sunglasses. A different group of participants were then asked to name either veridical or manipulated composites. These were named twice: firstly, when viewed from the front, and secondly, from the side, to create a 'perceptual' stretch.

Recall that inversion generally leads to the disruption of configural information whilst leaving feature information intact [14-17]. Blur, on the other hand, does the opposite: it generally leads to a concealment of feature information whilst leaving configural information intact [18,19]. Findings showed that inversion made EvoFITs less identifiable, but had no effect for PRO-fits. Blur had the opposite effect, with making PRO-fits difficult to identify, but not EvoFITs. The combination of inversion-and-blur led to a significant decrease 
in naming for both composite types. Therefore, findings of the current experiment show that whilst feature composites seem more accurate in terms of their individual facial features, holistic composites seem more accurate in terms of their configural information.

It would appear that differences in face construction between systems led to these findings. Witnesses concentrate more on configural than featural information when constructing composites with holistic systems, and concentrate more on featural than configural information when constructing composites with feature systems. This evidence leads to the conclusion that both types of systems could usefully benefit from techniques which improve the accuracy of featural information (for holistic systems) and configural information (for feature systems). This would ideally lead to an overall more-accurate composite likeness for both types of system.

Composites were also manipulated by adding sunglasses to conceal their eye region. Recall that past research demonstrated that poor-quality composites became more recognisable with sunglasses added, whilst good-quality composites became less recognisable [26]. In the current experiment, sunglasses led to a significant increase in identifiability of PRO-fits relative to veridical, which seems to imply that their eye region was of poor quality: their concealment made them more recognisable suggesting that their presence was disruptive. This further led to PRO-fits with sunglasses being significantly more recognisable than EvoFITs with sunglasses. This is an unusual result as EvoFIT composites have continuously been shown to lead to significantly higher identification than PRO-fit composites (e.g. [25,26]) - that is, one would expect EvoFIT composites to be accurately constructed around the eyes. As sunglasses did not decrease naming percentages in EvoFITs as originally hypothesised, it seems that their eye region was not necessary for recognition. The lack of decrease or increase of naming rates suggests that the eyes were neither accurate enough to enable recognition, nor inaccurate enough to be disruptive. Another explanation, however, would be poor experimental power as naming values were quite low overall. The trend for EvoFIT goes in the expected direction - that is, identification rates for EvoFITs with sunglasses declines-but fails to approach significance (see Fig. 2).

Findings further revealed that looking at veridical composites from the side helped identification. This beneficial effect replicates previous studies [23, 25]. Composites tend to be described as being of poor to average likeness [24], anddifferently to photographs - each facial part could potentially lack accuracy. Therefore, these inaccurate features might support each other, and may all be equally important for recognition due to the greater amount of facial cues available. This would also be in line with theories of holistic face processing [28,29]. Therefore, perceptually stretching composites might help in overcoming the inaccurate aspects which are naturally present in composites.
Incorrect naming of composites was also considered, to investigate the extent to which participants' were willing to offer a name for each composite. It was found that composites were incorrectly named at $8 \%$ overall which, in comparison to past studies (e.g. [25,27]) is rather low. This suggests that participants were reluctant to provide any name, which led to a knock-on effect of participants also providing fewer correct names. This is an issue with participant response-bias and seems to explain the somewhat low correct naming overall.

In conclusion, findings of our experiment seem valuable as they provide possible ways to increase the effectiveness of composites. In practice, the police could publish composites in a veridical form as well as with sunglasses - although it would be sensible to check this first experimentally. Also, people could be asked to look at the veridical composite from the side to help recognition. This procedure has already been trialled by the UK police and is now in regular use [23]. Both of these procedures could help increasing the likelihood of identification and arrest rates of perpetrators, and thereby maintain security.

\section{REFERENCES}

[1] C.C. Goren, M. Sarty, and P.Y.K. Wu, "Visual following and pattern discrimination of face-like stimuli by newborn infants," in Pediatrics, vol. 56, pp. 544-549, 1975.

[2] C. Jaques, and B. Rossion, "The speed of individual face categorization," in Psychological Science, vol. 17, pp. 485-492, 2006.

[3] R.A. Johnston, and A.J. Edmonds, "Familiar and unfamiliar face recognition: a review," in Memory, vol. 17, pp. 577-596, 2009.

[4] V. Bruce, and A. Young, In the Eye of the Beholder: The Science of Face Perception. New York: Oxford University Press, 1998.

[5] H.D. Ellis, J.W. Shepherd, and G.M. Davies, "Identification of familiar and unfamiliar faces from internal and external features: some implications for theories of face recognition," in Perception, vol. 8, pp. 431-439, 1979.

[6] C. O'Donnell, and V. Bruce, "Familiarisation with faces selectively enhances sensitivity to changes made to the eyes," in Perception, vol. 30, pp. 755-764, 2001.

[7] R. Cabeza, and T. Kato, "Features are also important: contributions of featural and configural processing to face recognition," in Psychological Science, vol. 11, pp. 429-433, 2000.

[8] G.J. Hole, "Configurational factors in the perception of unfamiliar faces," in Perception, vol. 23, pp. 65-74, 1994.

[9] J. Hochberg, and R.R. Galper, "Recognition of faces: an explanatory study," in Psychological Science, vol. 9, pp. 619-620, 1967.

[10] R.K. Yin, "Looking at upside-down faces," in Journal of Eperimental Psychology, vol. 81, pp. 141-145, 1969.

[11] T. Valentine, "Upside-down faces: a review of the effect of inversion upon face recognition," in British Journal of Psychology, vol. 79, pp. 471-491, 1988.

[12] V. Bruce, T. Doyle, N. Dench, and M. Burton, "Remembering facial configurations," in Cognition, vol. 38, pp. 109-144, 1991.

[13] R. Kemp, C. McManus, and T. Pigott, "Sensitivity to the displacement of facial features in negative and inverted faces," in Perception, vol. 9, pp. 531-543, 1990.

[14] A. Freire, K. Lee, and L.A. Symons, "The face-inversion effect as a deficit in the encoding of configural information: direct evidence," in Perception, vol. 29, pp. 159-170, 2000. 
[15] H. Leder, and V. Bruce, "When inverted faces are recognized: the role of configural information in face recognition," in Quarterly Journal of Experimental Psychology, vol. 53(A), pp. 513-536, 2000.

[16] B. Rossion, "Picture-plane inversion leads to qualitative changes of face perception," in Acta Psychologica, vol. 128, pp. 274-289, 2008.

[17] E. McKone, and R. Robbins, "Are faces special?," in The Oxford handbook of face perception, A.J. Calder, G. Rhodes, M.H. Johnson, and J.V. Haxby, Eds., Oxford, England: Oxford University Press, pp.149176, 2011.

[18] J. Sergent, "An investigation into component and configural processes underlying face perception," in British Journal of Psychology, vol. 75, pp. 221-243, 1984.

[19] G.J. Hole, and P.A. George, K. Eaves, and A. Rasek, "Effects of geometric distortions on face-recognition performance," in Perception, vol. 31, pp. 1221-1240, 2002.

[20] A. Schwaninger, J.S. Lobmaier, and S.M. Collishaw, "Role of featural and configurational information in familiar and unfamiliar face recognition," in Biologically Motivated Computer Vision, vol. 2525, pp. 643-650, 2002.

[21] S.M. Collishaw, and G.J. Hole, "Featural and configurational processes in the recognition of faces of different familiarity," in Perception, vol. 29, pp. 893-909, 2000.

[22] S.M. Collishaw, and G.J. Hole, "Is there a linear or a nonlinear relationship between rotation and configural processing of faces?," in Perception, vol. 31, pp. 287-296, 2002.

[23] C.D. Frowd, F. Skelton, G. Hepton, L. Holden, S. Minahil, M. Pitchford, A. McIntyre, and P.J.B. Hancock, "Whole-face procedures for recovering facial images from memory," in Science \& Justice, vol. 53, pp. 89-97, 2013.

[24] A.M. Lech, and R. Johnston, "The relative utility of verbal descriptions and facial composites in facial identifications," in International Journal of Bio-Science and Bio-Technology, vol. 3(3), pp. 1-16, 2011.

[25] C.D. Frowd, S. Jones, C. Fodarella, F. Skelton, J. Marsh, L. Greenwood, H. Khan, S. Fields, K. Kearly, A.H. McIntyre, and P.J.B. Hancock, "Configural and featural information in facial-composite images," in Ergonomics, under revision.

[26] A.H. McIntyre, P.J.B. Hancock, C.D. Frowd, and V. Bruce, "Seeing more clearly with glasses?: the impact of glasses and technology on unfamiliar face matching and identification of familiar facial composites," in International Conference on Emerging Security Technologies, pp. 50-55, 2010.

[27] C.D. Frowd, M. Pitchford, V. Bruce, S. Jackson, G. Hepton, M. Greenall, A. McIntyre, and P.J.B. Hancock, "The psychology of face construction: giving evolution a helping hand," in Applied Cognitive Psychology, vol. 25(2), pp. 195-203, 2011.

[28] T. Valentine, "A unified account of the effects of distinctiveness, inversion, and race in face recognition," in Quarterly Journal of Experimental Psychology - Human Experimental Psychology, vol. 43(A), pp. 161-204, 1991.

[29] T. Valentine, and M. Endo, "Towards an exemplar model of face processing - the effects of race and distinctiveness," in Quarterly Journal of Experimental Psychology - Human Experimental Psychology, vol. 44 (A), pp. 671-703, 1992 